\title{
Entre la vigne et la croix. Dionysos dans les discours littéraires et figurés chrétiens (IIe-IVe siècles)
}

Doctorat d'Histoire des Religions, sous la direction de Giovanni Filoramo, et Nicole Belayche, Fondazione San Carlo (Modène) et École pratique des hautes études (EPHE), soutenu le 23 juin 2011.

\section{Francesco Massa}

\section{(2) OpenEdition}

\section{Édition électronique}

URL : http://journals.openedition.org/assr/24593

DOI : $10.4000 /$ assr.24593

ISSN : $1777-5825$

Éditeur

Éditions de l'EHESS

\section{Édition imprimée}

Date de publication : 30 décembre 2012

Pagination : 309-358

ISSN : 0335-5985

\section{Référence électronique}

Francesco Massa, «Entre la vigne et la croix. Dionysos dans les discours littéraires et figurés chrétiens (IIe-Ive siècles) ", Archives de sciences sociales des religions [En ligne], 160 | octobre-décembre 2012, mis en ligne le 14 mars 2013, consulté le 02 mai 2019. URL : http://journals.openedition.org/ assr/24593

Ce document a été généré automatiquement le 2 mai 2019.

(C) Archives de sciences sociales des religions 


\section{Entre la vigne et la croix. Dionysos dans les discours littéraires et figurés chrétiens (IIe-IVe siècles) ${ }^{1}$}

Doctorat d'Histoire des Religions, sous la direction de Giovanni Filoramo, et Nicole Belayche, Fondazione San Carlo (Modène) et École pratique des hautes études (EPHE), soutenu le 23 juin 2011.

Francesco Massa

1 Mon doctorat est consacré à l'analyse du rôle du dieu grec Dionysos dans la production littéraire et figurée chrétienne des premiers siècles de l'époque impériale romaine ( $\mathrm{II}^{\mathrm{e}}-\mathrm{IV} \mathrm{e}^{\mathrm{e}}$ siècles). La recherche s'inscrit dans deux courants d'études principaux: le premier renouvelle l'analyse des contacts religieux pendant les premiers siècles de notre ère, en proposant d'éclairer comment les situations de contact interviennent dans la formation et la définition des identités religieuses; le deuxième inscrit le christianisme ancien au nombre des composantes à part entière des religions de l'Empire romain, par-delà les considérations doctrinales et théologiques.

2 Entre les II ${ }^{\mathrm{e}}-\mathrm{IV}{ }^{\mathrm{e}}$ siècles, les récits mythiques, les pratiques rituelles et les représentations iconographiques dionysiaques ont constitué des éléments de rencontre importants avec les groupes chrétiens présents dans les territoires de l'Empire. Le processus de sélection mis en œuvre par les sources littéraires et figurées chrétiennes sur les représentations dionysiaques transparaît, d'un côté, à travers l'identification de traits qui ont permis la superposition et la reconfiguration des éléments dionysiaques du point de vue chrétien, et de l'autre côté, à travers l'étude des modalités et des instruments d'appropriation et/ ou de neutralisation des images et du vocabulaire dionysiaques.

Dans cette perspective, il a semblé nécessaire de dépasser la question des rapports entre cultes à mystères et religion chrétienne et d'abandonner les théories de dérivation ou les comparaisons généalogiques entre les deux formes religieuses. Il nous a paru plus utile de revenir aux sources et aux documents anciens et de souligner qu'il a existé, pendant les siècles de l'Antiquité tardive, des aspects qui permettaient de comparer de manière 
explicite Dionysos et Jésus. Un point de départ d'autant plus valide que ce sont les chrétiens eux-mêmes qui ont reconnu ces analogies.

4 Afin d'identifier les différentes phases de la rencontre entre Dionysos et les chrétiens et de mettre en évidence les réponses spécifiques que les textes et les images chrétiennes ont opposées aux représentations dionysiaques qui pouvaient évoquer des ressemblances avec le christianisme, nous avons isolé trois modèles opératoires chrétiens face aux rites, aux mythes et aux images liés à Dionysos :

5 - identification des analogies, dans un but apologétique, ainsi que leur justification ou/et leur négation, d'un point de vue polémique : une double attitude, défensive et offensive, qui utilise des théories communes et répandues comme celle de l'imitatio diabolica ;

6 - processus de sélection et d'utilisation de mots et d'images dionysiaques, de la part d'auteurs et de commanditaires chrétiens: un phénomène que l'on pourrait qualifier d'« emprunt », parce qu'il entraîne un processus d'appropriation et de resémantisation partielle des mots et des images dionysiaques;

7 - «Interpretatio » chrétienne de thèmes et de motifs dionysiaques : à travers un processus de médiation culturelle qui emprunte parfois la voie de l'allégorie; les auteurs mènent une large opération de reprise et de transformation des scénarios dionysiaques (littéraires ou figurés), d'un point de vue chrétien.

8 La grille à travers laquelle nous avons choisi de lire et de rassembler les témoignages dont nous disposons n'est qu'un instrument opératoire qui permet de mettre en évidence la variété des prises de position exprimées par les textes et les images chrétiennes. Selon les périodes et sans doute davantage encore selon les lieux et les contextes, les auteurs chrétiens se sont comportés très différemment. Ils étaient cependant complètement insérés dans le contexte culturel gréco-romain et sa paideia : ils partageaient un savoir largement répandu parmi les personnalités intellectuelles de l'époque et produisaient des textes dont les modèles et les formes littéraires dérivaient de la tradition précédente.

9 La cité d'Alexandrie, lieu de compétition religieuse et intellectuelle, centre par excellence de la cohabitation religieuse dès la période hellénistique, représente tout particulièrement la patrie d'élection de la rencontre entre le monde dionysiaque et le monde chrétien. L'opération intellectuelle la plus intéressante et la plus complexe sur les représentations dionysiaques, du point de vue littéraire et religieux, a été accomplie par Clément d'Alexandrie (fin II $^{\mathrm{e}}$ siècle - début ${ }^{\mathrm{e}}{ }^{\mathrm{e}}$ siècle), dans le Protreptique aux Hellènes et les Stromates. La stratégie de récupération et de resémantisation de la matière dionysiaque, chez Clément, est extrêmement transparente, car elle est, en grande partie, construite sur la citation et sur l'évocation des Bacchantes d'Euripide. Son écriture est construite à travers un processus de sélection et d'appropriation du vocabulaire et des images qui traditionnellement étaient liés à Dionysos. La pièce euripidéenne mettait en scène l'épiphanie d'un dieu, né d'une femme mortelle, qui décide de prendre la morphé, la forme humaine, et de revenir à sa terre d'origine pour fonder son nouveau culte et pour être reconnu comme dieu. Mais tout d'abord, son culte n'est pas accepté par le pouvoir politique de la cité grecque, et le souverain de Thèbes, Penthée, ordonne d'emprisonner cet étranger et son cortège de bacchantes. Dans la situation historique du christianisme naissant, l'intrigue euripidéenne, qui met en scène l'adhésion - volontaire ou imposée - aux nouveaux rites de Dionysos, pouvait bien sûr représenter un scénario invitant à une possible comparaison avec la thématique de la "conversion» et les conséquences dérivées du refus à l'égard du nouveau culte. Dans cette perspective, 
Clément évoque deux personnages d'Euripide, Penthée et Tirésias, en les invitant à la conversion et en soulignant la grande différence qui passait entre le Dionysos impitoyable de la tragédie et le nouveau dieu des chrétiens.

Cependant, l'intérêt que Clément montre à l'égard des cultes dionysiaques ne s'épuise pas en évoquant la scène des Bacchantes d'Euripide: l'auteur trace ensuite un nouveau scénario chrétien, qui est représenté à travers un usage filtré du lexique dionysiaque. Sélectionnant un certain nombre de termes spécifiques à ce langage, Clément décrit un paysage édénique représentatif par certains aspects d'un milieu de culte alternatif au mont Cithéron, mais qui conserve d'un autre côté des traits spécifiques au culte de Dionysos. Sur ce mont sobre, dédié aux véritables représentations, les filles de Dieu font les bacchantes (bakcheuousi) : de plus, les fidèles font partie d'un thiase et célèbrent les " rites du Verbe », nommés orgia, un terme spécifique des cultes dionysiaques. L'écriture de Clément est construite à travers un processus de sélection et d'appropriation du vocabulaire et des images qui étaient traditionnellement liés à Dionysos. L'auteur est bien conscient que le nouveau message chrétien ne peut pas être accepté sans un processus de traduction dans les catégories de la culture grecque. Conscient des analogies que les païens pouvaient trouver entre mystères dionysiaques et mystères chrétiens, Clément ne cherche pas à les cacher ou à les attribuer à l'intervention des daimones; au contraire, il utilise ces analogies à son avantage, il les assume, en créant un langage plus persuasif à l'égard des destinataires de son ouvrage.

11 Pour mettre en évidence les résultats de cette recherche, il faut considérer en premier les cas où c'est le dieu grec en personne qui joue un rôle principal dans les rencontres entre Dionysos et les chrétiens : Justin (milieu du II ${ }^{\mathrm{e}}$ siècle) compare Dionysos et Jésus, leurs biographies de «fils de dieu », en traçant une «carte» des analogies dangereuses entre les deux divinités. De son côté, Firmicus Maternus (première moitié du IV siècle) part du même principe - c'est-à-dire de l'identification d'un certain nombre d'analogies -, mais dans le but de prononcer une condamnation radicale de Dionysos, se concentrant sur la superposition et l'assimilation avec le Diable : la situation historique du IV siècle, en fait, conduisait l'auteur à invoquer la destruction des cultes païens, considérés comme un produit du Diable. Clément d'Alexandrie utilise les mots du Dionysos des Bacchantes d'Euripide également pour illustrer une scène d'initiation chrétienne où Jésus est le mystagogos, en exploitant les analogies entre les deux figures divines afin de montrer que Jésus est le vrai dieu et le rituel chrétien le vrai rituel. En ce qui concerne les représentations figurées, de manière analogue, les représentations de Jésus - la chevelure flottante et les traits délicats du visage - renvoient aux figures divines des dieux des mondes grec et romain, parmi lesquels il faut considérer Dionysos; le même discours est possible pour les figurations de Dionysos sur la croupe d'un âne, dans le schéma iconographique de l'entrée à Jérusalem, ou bien pour la représentation de l'enfance du dieu, la typologie de la "Vierge à l'Enfant», et la représentation de l'« Adoration des Mages ».

12 Pourtant cette comparaison entre Dionysos et Jésus n'épuise pas le problème de leur rencontre tout au long de l'époque impériale. Souvent, d'autres éléments des représentations bacchiques ont conduit les chrétiens à sélectionner des images et des mots dionysiaques pour la construction de nouveaux discours chrétiens. Nous avons vu par exemple dans le Contre Celse d'Origène les modalités de la représentation de la vie après la mort ; ou la définition des cérémonies chrétiennes par le terme dionysiaque orgia ; l'attribution de la mania bacchique à des figures chrétiennes ; l'organisation des groupes 
des fidèles, comme le montre l'usage du terme thiasos, du moins dans des contextes littéraires de Clément d'Alexandrie, d'Origène et d'Eusèbe de Césarée; ou encore la reprise des modalités de représentation des fêtes bacchiques et des fêtes chrétiennesdans les homélies de Jean Chrysostome, qui transfère sur les pratiques chrétiennes des éléments de la joie et de l'enthousiasme des cortèges dionysiaques.

Enfin, il faut tenir compte du thème important concernant le vin, la vigne et la vendange, qui traverse différentes typologies de documents, différents lieux et temps, en soulignant une récupération et une resémantisation du patrimoine bacchiques. En général, les images nous montrent que les chrétiens ont utilisé l'écho dionysiaque qui résonnait dans l'allégorie de la vigne pour diffuser la conception d'un dieu qui concentrait sur sa propre figure des thèmes et des aspects de la production et de l'usage du vin. En effet, bien que le vin soit dans les textes et les images chrétiens le résultat de traditions culturelles et religieuses diverses, avant tout la tradition juive, il me semble que l'on peut soutenir que sans Dionysos et ses traditions, le vin des chrétiens aurait probablement assumé des formes et des significations en partie différentes.

L'analyse des sources permet de souligner que la rencontre entre Dionysos et les discours chrétiens n'a pas engendré un simple phénomène de reprise : en effet, nous ne pouvons pas parler exclusivement d'un processus de «continuité ». Les discours littéraires et figurés dionysiaques ont été l'objet d'un processus de sélection et de nouvelle définition de la part des images et des textes chrétiens. L'analyse des représentations dionysiaques dans les images et les textes chrétiens nous a permis d'éclairer un aspect de la complexité des cohabitations et contacts religieux et de mettre en évidence le rôle des représentations dionysiaques dans le processus de formation de l'identité chrétienne.

\section{NOTES}

1. À paraître chez Franz Steiner, Stuttgart.

\section{AUTEUR}

FRANCESCO MASSA

f.massa@tin.it 